Bayero Journal of Pure and Applied Sciences, 10(1): 20 - 27

ISSN 2006 - 6996

\title{
ANALYSIS OF THE PROBABILITY OF CHANNEL SATISFACTORY STATE IN P2P LIVE STREAMING SYSTEMS
}

\author{
${ }^{1}$ Adamu, A. and ${ }^{2}$ Ahmed, J. B. \\ ${ }^{1}$ Umaru Musa Yar'adua University, Katsina State, Nigeria. \\ Mathematics and Computer Science Department. \\ ${ }^{2}$ Federal University, Dutsin-ma, Katsina State, Nigeria. \\ Mathematical Sciences and IT Department. \\ 1'aminu@mail.ru: +2348031581658; ${ }^{2}$ bjamilu@fudutsinma.edu.ng: +2347067712786
}

\begin{abstract}
In this paper a model based on user behaviour of P2P live streaming systems was developed in order to analyse one of the key QoS parameter of such systems, i.e. the probability of channel-satisfactory state, the impact of upload bandwidths and channels' popularity on the probability of channel-satisfactory state was also analysed. Results obtained have shown that channels with high number of patronising peers achieve satisfactory state easily while channels with low number of peers hardly attain satisfactory state. This called for the need to design a framework or incentive scheme for effective functionality of $P 2 P$ live streaming systems which will favour all channels regardless of their popularities.
\end{abstract}

Keywords: P2P Systems, QoS Parameters, Satisfactory State, Video Streaming, Fluid Model.

\section{INTRODUCTION}

Generally, P2P systems are tree-based or meshbased according to the overlay construction of peers; unstructured or structured according to the ability of peers to easily locate their desired chunks from other neighbouring peers in the network and are functionally used for file sharing, on-demand and live video streaming (Adamu and Bashir, 2015). Services in P2P systems are provided using P2P model, where each user in the system can provide and consume resources, in other word can act as server and client simultaneously and in this case a user is called a peer (unlike the client server model where all the resources come from the server only)(Shen, Yu, Buford \& Akon, 2010). There are multiples of successfully deployed P2P file sharing systems such as Bit Torrent, $\mu$ Torrent, Napster, etc., likewise, there are many successfully deployed P2P streaming systems where video services are provided on real-time and on demand basis using P2P model e.g.PPLive, PPStream, SopCast, TVAnts, UUSee, etc. This paper considers only the P2P live streaming systems. Similar to traditional Cable TV systems, P2P streaming systems provide peers with multiple channels, peers viewing the same channel are grouped together to form a swarm for that channel sharing and viewing the stream of the channel. Each $m$-channel in the system has its defined streaming rate $R_{m}$ in Kbps with which peers should view the channel without any quality degradation. When all participating peers viewing $m$-channel are receiving the video stream of $m$-channel at a rate greater than or equal to $R_{m} \mathrm{Kbps}$, then $m$-channel and its viewers are said to be in satisfactory state.

This paper analyses the probability of channelsatisfactory state as one of the key Quality of Service (QoS) parameter for P2P live streaming systems, unlike the models developed in Ross, Kumar and Liu (2007) as well as Ross, Liu and Wu (2010), the model developed in this paper takes into account user behaviour, i.e. peer churn and bits flow was modelled as fluid flow. The applicability of the theory of probability was deduced from Kelly (1991).

Section II of the paper provides the model of P2P live streaming systems taking into account peer behaviour and expression was obtained for the computation of the probability of channelsatisfactory state. In section III, analysis of the model was conducted with heterogeneous peers in terms of upload rates. A case study consisting of 100 channels and 2000 peers is numerically analysed in section IV. Section V concludes the paper. 


\section{MODEL OF P2P LIVE STREAMINGSYSTEMBASED ON USER BEHAVIOUR}

Let's consider a P2P live streaming system with multiple channels $|\mathrm{M}|=M$ web casted to peers. In the system there are $|\mathrm{N}|=N$ peers, each one of them is viewing one of the channels, the popularity of the $m$-channel is denoted by $\rho_{m}, \sum_{m=1}^{M} \rho_{m}=1$ and the average viewing time of a $m$ channel by $\mu_{m}^{-1}$.

Assume that peers freely switch from one channel to another, and they do that independently. Denote by $x_{n m} \in\{0,1\}$ the state of $n$-peer on $m$-channel, $x_{n m}=1$, if $n$-peer is viewing $m$ channel, else $x_{n m}=0$. The state of the entire system is given by a matrix $\mathbf{X}=\left(x_{n m}\right)_{n \in \mathrm{N}, m \in \mathrm{M}}$ and state space of the system is

$$
\mathrm{X}=\left\{\mathbf{X}: x_{n m} \in\{0,1\}, \sum_{m \in \mathrm{M}} x_{n m}=1, n \in \mathrm{N}\right\} \text {. }
$$

Note that in the matrix $\mathrm{X}$, the sum $x_{m}=\sum_{n \in \mathrm{N}} x_{n m}$ on $m$-column corresponds to the number of peers viewing $m$-channel, when the system is in state $X$. The state space of the system when exactly kpeers are viewing $m$-channel has the form

$$
\mathrm{X}_{m}(k)=\left\{\mathbf{X} \in \mathbf{X}: x_{m}=k\right\}, k=\overline{0, N}, m \in \mathrm{M} \text {. (2) }
$$

Then the marginal distribution $p_{m}(k)$ of the number of peers viewingm-channel is given by the formula below

$$
p_{m}(k)=P\left\{\mathbf{X} \in \mathbf{X}_{m}(k)\right\}=\sum_{\mathbf{X}=\mathbf{X}_{m}(k)} P(\mathbf{X}), k=\overline{0, N}, m \in \mathrm{M}_{(3)}
$$

Since channels and peers are independent of one another and their behaviors are independent, then we can have the following proposition.

Proposition 1. The probability $P(\mathbf{X})$ that a system with $N<\infty$ peers is in state $\mathrm{X}$ is defined by the formula

$$
P(\mathbf{X})=\prod_{n \in \mathrm{N}} \prod_{m \in \mathrm{M}} \rho_{m}^{x_{n m}}(N), \mathbf{X} \in \mathbf{X} .
$$

Proposition 2.For a system with $N<\infty$ peers, the marginal distribution of the number of peers viewing $m$-channel is

$p_{m}(k)=\left(\begin{array}{c}N \\ k\end{array}\right) \rho_{m}^{k}(N)\left(1-\rho_{m}(N)\right)^{N-k}, k=\overline{0, N}, m \in \mathrm{M}$.

InP2P live streaming systems, video stream distribution is done via P2P model, then the video stream obtained by a peer viewing $m$-channel comes both from the video server and the neighboring peers viewing the same $m$-channel. Denote by $s_{m}$ the upload bandwidth of the videoserver dedicated for web casting the stream of $m$-channel, and $u_{n}$ upload bandwidth of $n$-peer devoted to the system.

Denote by $R_{m}$ the playback rate of $m$-channel, i.e. the required rate to view $m$-channel without pauses, with synchronized image and sound, and without all other phenomenon that can deteriorate the peer viewing quality. Assume the system is in state $\mathbf{X}$, then the value $w_{n m}(\mathbf{X})$ denote the available video stream to $n$-peer viewing $m$-channel. To playback $m$-channel with a standard quality, $n$-peer must obtain video stream of $m$-channel from the system at rate not less than $R_{m}$, i.e. $w_{n m}(\mathbf{X}) \geq R_{m}$ (Figure 1 ). 


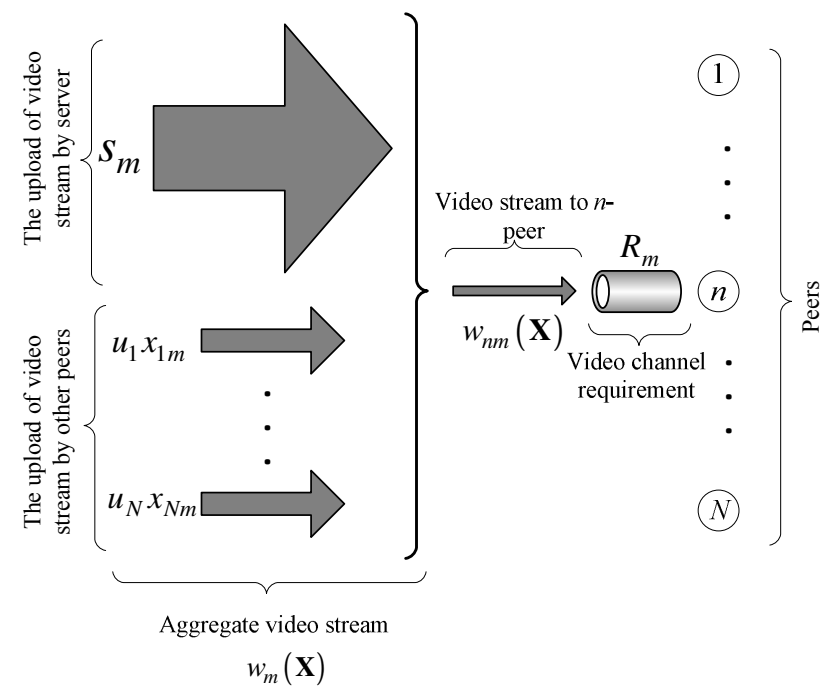

Figure 1.The scheme of video formation in P2P live streaming system

Then the value $w_{m}(\mathbf{X})$ will be the sum of all video streams available to all peers viewing $m$ channel when the system is in $\mathbf{X}$ state, and it can be expressed in the following form

$$
w_{m}(\mathbf{X})=\sum_{n \in \mathrm{N}} w_{n m}=s_{m}+\sum_{n \in \mathrm{N}} x_{n m} u_{n} \text { (6) }
$$

Definition 1.m-channel is said to be in a satisfactory state, if all peers viewing the channel are receiving video stream at rate not less than the channel rate $R_{m}$.

If the inequality $w_{m}(\mathbf{X}) \geq R_{m} \sum_{n \in \mathrm{N}} x_{n m}$ holds, then condition for $m$-channel to be in satisfactory state is fulfilled.

Denote by $A_{m}$ an event in which $m$-channel is viewed by peers at the required rate $R_{m}$.

$$
\mathrm{A}_{m}=\left\{\mathbf{X} \in \mathrm{X}: s_{m}+\sum_{n \in \mathrm{N}} x_{n m} u_{n} \geq R_{m} \sum_{n \in \mathrm{N}} x_{n m}\right\} \text { (7) }
$$

And by $\pi_{m}$ the probability of the event in (7), subsequently, $\pi_{m}$ will be the probability that $m$-channel is in satisfactory state;

$$
\pi_{m}=P\left(\mathrm{~A}_{m}\right)=\sum_{\mathbf{X} \in \mathrm{X}} \mathrm{I}\left(\mathrm{A}_{m}\right) P(\mathbf{X})
$$

Where $I\left(A_{m}\right)$ is a unit function:

$$
\mathrm{I}\left(\mathrm{A}_{m}\right)=\left\{\begin{array}{l}
1, \text { if event } \mathrm{A}_{m} \text { holds; } \\
0, \text { otherwise. }
\end{array}\right.
$$

The probability that $m$-channel of the considered system is in satisfactory state is defined by formula

$$
\pi_{m}=\sum_{\mathbf{X} \in \mathrm{X}} \mathrm{I}\left(\mathrm{A}_{m}\right) P(\mathbf{X})=\sum_{\mathbf{X} \in \mathrm{X}} \mathrm{I}\left(\mathrm{A}_{m}\right) \prod_{n \in \mathbb{N}} \prod_{n \in \mathbb{M}} \rho_{m}^{x_{m}}(N) \text { (9) }
$$

In the next section an analysis of the defined QoS parameter would be conducted for a system with two categories of peers, i.e. with high and low upload bandwidth.

\section{ANALYSIS OF THE MODEL WITH TWO CATEGORIES OF PEERS}

For the analysis, assume that in the system there are two categories of peers: peers with high upload bandwidth and peers with low upload bandwidth. Assume also peers with high upload bandwidth have equal upload bandwidth $u^{h}$ and peers with low upload bandwidth have equal upload bandwidth $u^{l}$, where $u^{l}<u^{h}$, and the corresponding subset of peers with these upload bandwidths are denoted by $\mathrm{N}^{h}$ and $\mathrm{N}^{l}$ respectively. In this case $N^{h}=\left|\mathrm{N}^{h}\right|, N^{l}=\left|\mathrm{N}^{l}\right|$ and $\mathrm{N}=\mathrm{N}^{h} \cup \mathrm{N}^{l}$.

Then

$u_{n}=\left\{\begin{array}{l}u^{h}, n \in \mathrm{N}^{h}, \\ u^{l}, n \in \mathrm{N}^{l} .\end{array}\right.$

In line with earlier introduced parameters, in each system state $\mathrm{X}$, the value $x_{m}^{h}=\sum_{n \in \mathrm{N}^{h}} x_{n m}$ denotes the number of peers on $m$-channel with high upload bandwidth and $x_{m}^{l}=\sum_{n \in \mathrm{N}^{l}} x_{n m}$ the number of peers on $m$ channel with low upload bandwidth and in this case $x_{m}=x_{m}^{h}+x_{m}^{l}, m \in \mathrm{M}$. Then the event $A_{m}$ from (7) will have the following form. 


$$
\mathrm{A}_{m}=\left\{\left(x_{m}^{h}, x_{m}^{l}\right): 0 \leq x_{m}^{h} \leq N^{h}, 0 \leq x_{m}^{l} \leq N^{l}, s_{m}+x_{m}^{h} u^{h}+x_{m}^{l} u^{l} \geq x_{m} R_{m}\right\}
$$

Hence, the following proposition holds.

Proposition 3.For a P2P live streaming system with a finite number of peers with high and low upload bandwidth, the probability that $m$-channel is in satisfactory state is defined by the formula (11)

$\pi_{m}=P\left(\mathrm{~A}_{m}\right)=\sum_{x_{m}^{h}=0}^{N^{h}} \sum_{x_{m}^{l}=0}^{N^{l}} \mathrm{I}\left(\mathrm{A}_{m}\right) p_{m}\left(x_{m}^{h}\right) p_{m}\left(x_{m}^{l}\right), m \in \mathrm{M}$,

Where the marginal distribution of $p\left(x_{m}^{s}\right), s \in\{h, l\}$ has the form (12)

$$
p_{m}\left(x_{m}^{s}\right)=\left(\begin{array}{c}
N^{s} \\
x_{m}^{s}
\end{array}\right) \rho_{m}^{x_{m}^{s}}\left(N^{s}\right)\left(1-\rho_{m}\left(N^{s}\right)\right)^{N^{s}-x_{m}^{s}}, x_{m}^{s}=\overline{0, N^{s}}, m \in \mathrm{M}, s \in\{h, l\} \text {. }
$$

Proposition 4. For a P2P live streaming system with infinite number of peers with high and low upload bandwidth, the probability that $m$-channel is in satisfactory state is defined by the formula (13)

$$
\pi_{m}=P\left(\mathrm{~A}_{m}\right)=\sum_{x_{m}^{h}=0}^{\infty} \sum_{x_{m}^{l}=0}^{\infty} \mathrm{I}\left(\mathrm{A}_{m}\right) p_{m}\left(x_{m}^{h}\right) p_{m}\left(x_{m}^{l}\right), m \in \mathrm{M},
$$

Where the marginal distribution of $p\left(x_{m}^{s}\right), s \in\{h, l\}$ has the form

$$
\begin{aligned}
& p_{m}\left(x_{m}^{s}\right)=e^{-\gamma_{m}^{s}} \cdot \frac{\left(\gamma_{m}^{s}\right)^{x_{m}^{s}}}{x_{m}^{s} !}, m \in \mathrm{M}, s \in\{h, l\}, \\
& \gamma_{m}^{s}=\lim _{N^{s} \rightarrow \infty} N^{s} \rho_{m}\left(N^{s}\right), m \in \mathrm{M}, s \in\{h, l\} .
\end{aligned}
$$

\section{CASE STUDY}

For the case study, a P2P live streaming system with $M=100$ channels webcasted to the population of peers $N=2000$ was considered, each of which is viewing one of the channels. Assume the popularity of channels is distributed according to Zip $f$ distribution with parameter $z=1$, i.e. $\rho_{m}=\left(m^{z} \sum_{m=1}^{M} \frac{1}{m^{z}}\right)^{-1}, m \in \mathrm{M}$, in this case channels are numbered in descending order of popularities. All the channels have equal streaming rate, $R_{m}=R=500, \mathrm{Kbps}, m \in \mathrm{M}$.Assume $N^{l}=0.5 N$ peers have low upload bandwidth, the rest have high upload bandwidth. Peer with high upload bandwidth distribute video stream at rate $u^{h}=1500 \mathrm{Kbps}$ and peers with low upload bandwidth at rate $u^{l}=100 \mathrm{Kbps}$.

The graph in Figure 2 shows that the probability of channel satisfactory state for the first six (6) channels is 1 , whereas for the least popular channel (100-channel), the probability of satisfactory state is $\mathbf{0 . 8 7 9}$. This literally means that the probability of channel satisfactory state decreases as the channels' popularities decreases.

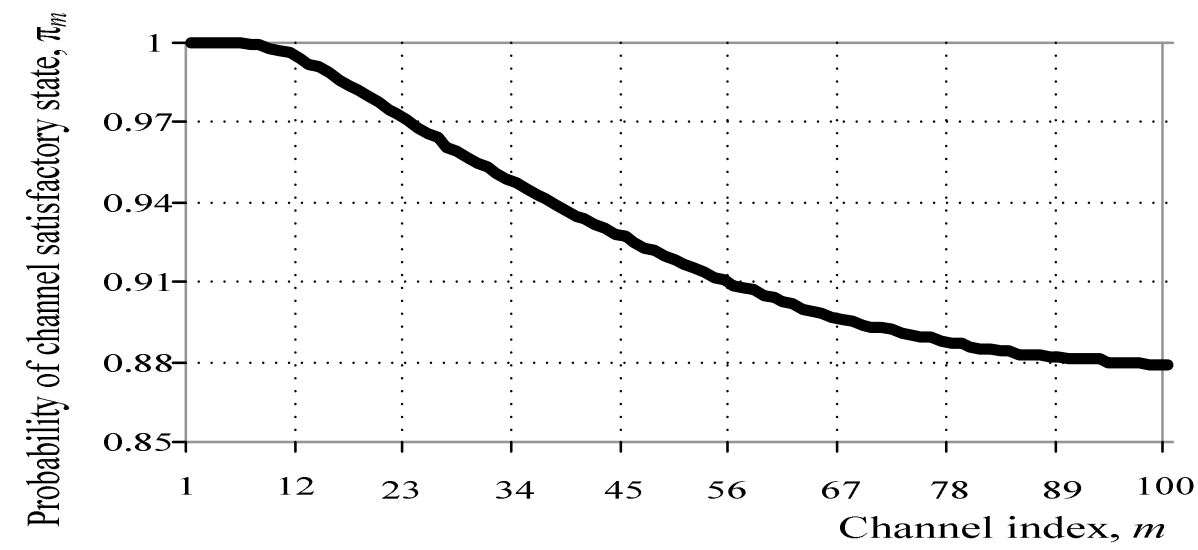

Figure 2. Probability of channel satisfactory state vs. channels' popularities 
It would be interesting to conduct the analysis of the probability of channel satisfactory state with various combinations of the number of peers (with high and low upload bandwidth). For that, the graphs in Figure 3 and Figure 4 show how the probability $\pi_{m}$ changes based on the changes in the values of $N^{h}$ and $N^{l}$ for 1 channel and 100-channel, i.e. for channel with the least popularity and a channel with the highest popularity.

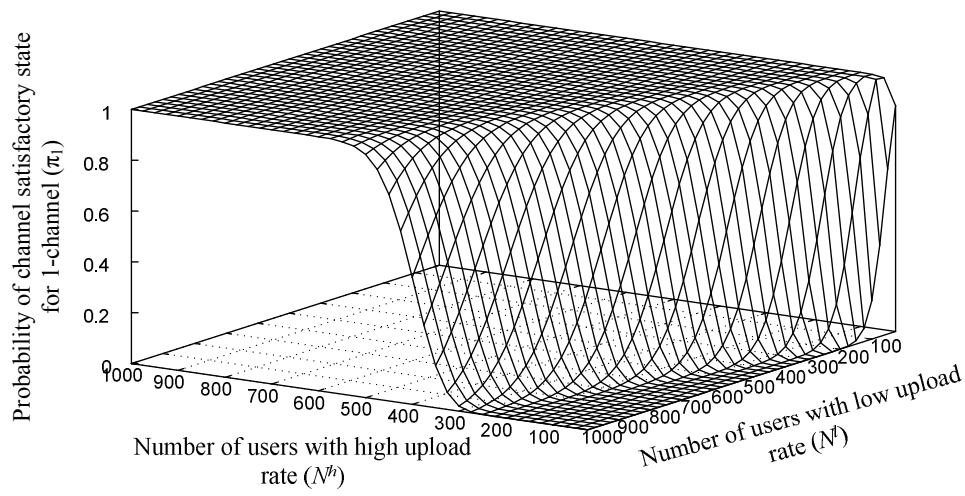

Figure $3 . \pi_{1}$ vs. $N^{h}$ and $N^{l}$

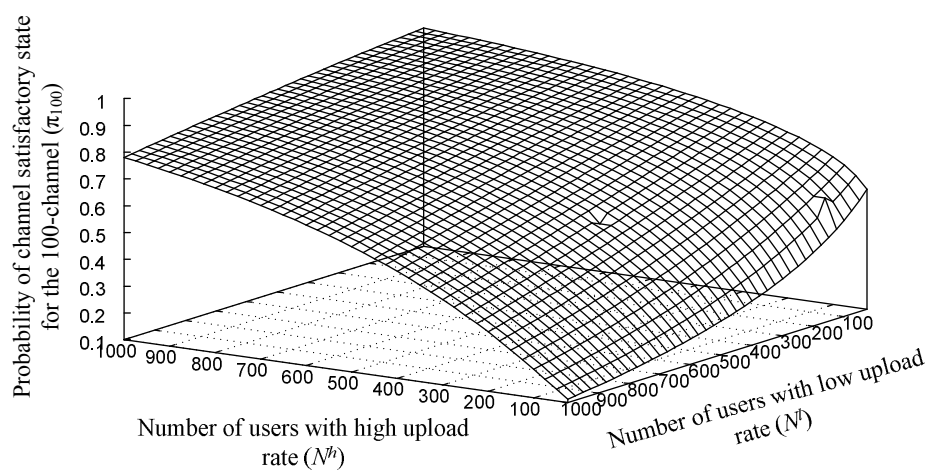

Figure $4 . \pi_{100}$ vs. $N^{h}$ and $N^{l}$

From the graph in Fig.3, it can be seen that the probability of channel satisfactory state for 1 channel quickly approaches 1 , even with few number of peers with high upload bandwidth. On the other hand, the graph in Figure 4 grows slowly and approaches 1 only when the population of peers with high upload bandwidth dominated that of peers with low upload bandwidth.
The above analysis is confirmed by graph in Figure 5, which shows the dependency of the probability of channel satisfactory state on value $K=N^{h} \cdot\left(N^{l}\right)^{-1}$, i.e. the ratio of the number of peers withhigh upload bandwidth to number of peers with low upload bandwidth.

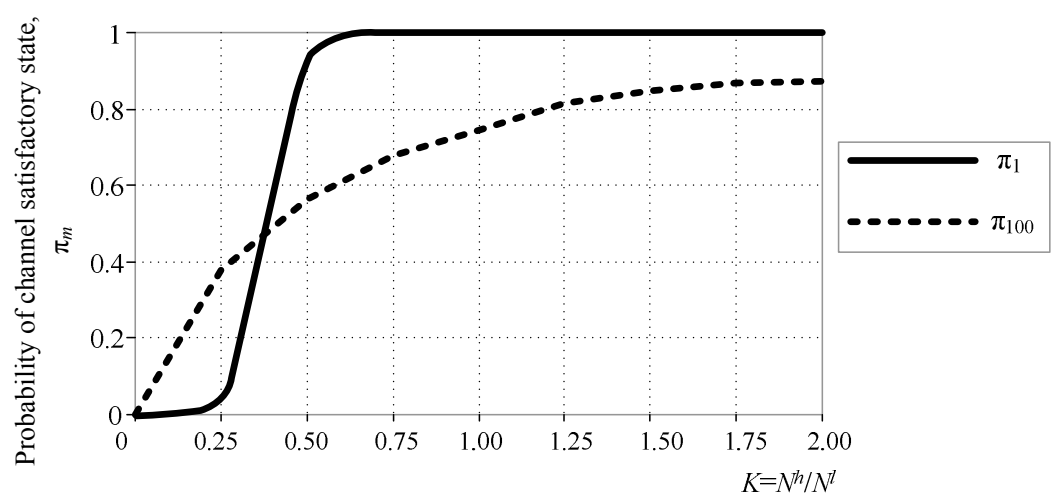

Figure $5 . \pi_{m}$ vs. $K$ 
It can be seen that the probability of channel satisfactory state approaches 1 for 1 -channel when $K \geq 0.5$, whereas the least popular channel (100-channel), even when $K=2$ the value of the probability does not exceed 0.9 .

It should be noted that the computation of probability $\pi_{m}$ with formula (11) is associated with computational difficulties for large values of $\mathrm{N}$, for that let's derive an approximation formula to ease the computation, to this end, let $\xi_{m}^{h}$ and $\xi_{m}^{l}$ be the random variables denoting the number of active high and low upload peers on m-channel respectively, $0 \leq \xi_{m}^{h} \leq N^{h}, 0 \leq \xi_{m}^{l} \leq N^{l}$. Assume that random variables $\xi_{m}^{h}$ and $\xi_{m}^{l}$ are distributed according to Poisson distribution with parameters $\gamma_{m}^{h}$ and $\gamma_{m}^{l}$ respectively,

$$
\begin{aligned}
& \gamma_{m}^{h}=\lim _{N^{h} \rightarrow \infty} N^{h} \rho_{m}\left(N^{h}\right), \\
& \gamma_{m}^{l}=\lim _{N^{l} \rightarrow \infty} N^{l} \rho_{m}\left(N^{l}\right) .
\end{aligned}
$$

Where $\gamma_{m}^{h}$ and $\gamma_{m}^{l}$ are the average number of peers with high and low upload bandwidth on $m$ channel (when there are $N^{h}$ and $N^{l}$ peers in the system) respectively.

Now let $K=\gamma_{m}^{h} / \gamma_{m}^{l}$ be the ratio of the average number of peers with high upload bandwidth on $m$-channel to the average number of peers with low upload bandwidth on $m$-channel. Let's rewrite the event $A_{m}$ as follows

$$
\begin{aligned}
& \mathrm{A}_{m}=\left\{\left(\xi_{m}^{h}, \xi_{m}^{l}\right): s_{m}+\xi_{m}^{h} u^{h}+\xi_{m}^{l} u^{l} \geq\left(\xi_{m}^{h}+\xi_{m}^{l}\right) R_{m}\right\}=\left\{\left(\xi_{m}^{h}, \xi_{m}^{l}\right): \xi_{m}^{h} \geq \varepsilon_{m} \xi_{m}^{l}-\delta_{m}\right\}, \\
& \mathrm{A}_{m}=\left\{\left(\xi_{m}^{h}, \xi_{m}^{l}\right): \xi_{m}^{h} \geq \varepsilon_{m} \xi_{m}^{l}-\delta_{m}\right\}, 0 \leq \xi_{m}^{h} \leq N^{h}, 0 \leq \xi_{m}^{l} \leq N^{l}, \\
& \text { Where } \varepsilon_{m}=\frac{R_{m}-u^{l}}{u^{h}-R_{m}} \text { and } \delta_{m}=\frac{S_{m}}{u^{h}-R_{m}} \text {. }
\end{aligned}
$$

Lemma1. Probability $\pi_{m}$ that $m$-channel is in satisfactory state for a system with infinite number of peers with high and low upload bandwidth can be approximated with normal distribution

$$
\begin{aligned}
& N\left(0, \frac{\gamma_{m}^{h}}{\gamma_{m}^{l}}+\varepsilon_{m}^{2}\right), \text { i.e. } \\
& \pi_{m}=\Phi\left(\frac{d_{m}}{\sqrt{K+\varepsilon_{m}^{2}}}\right),
\end{aligned}
$$

Where $\Phi(x)=\frac{1}{\sqrt{2 \pi}} \int_{-\infty}^{x} e^{-y^{2} / 2} d y$,

$$
d_{m}=\frac{\left(K-\varepsilon_{m}\right) \gamma_{m}^{l}+\delta_{m}}{\sqrt{\gamma_{m}^{l}}} \text { and } K=\frac{\gamma_{m}^{h}}{\gamma_{m}^{h}} \text {. }
$$

Proof: Let's normalize random variables $\xi_{m}^{h}$ and $\xi_{m}^{l}$, as follows:

$$
Z_{m}^{h}=\frac{\xi_{m}^{h}-\gamma_{m}^{h}}{\sqrt{\gamma_{m}^{h}}} \text { and } Z_{m}^{l}=\frac{\xi_{m}^{l}-\gamma_{m}^{l}}{\sqrt{\gamma_{m}^{l}}} \text {. }
$$

Considering (18), the event $A_{m}$ from (16) will take the form

$$
\mathrm{A}_{m}=\left\{\left(\xi_{m}^{h}, \xi_{m}^{l}\right): \xi_{m}^{h} \geq \varepsilon_{m} \xi_{m}^{l}-\delta_{m}\right\}=\left\{\sqrt{\gamma_{m}^{h}} Z_{m}^{h}+\gamma_{m}^{h} \geq \varepsilon_{m}\left(\sqrt{\gamma_{m}^{l}} Z_{m}^{l}+\gamma_{m}^{l}\right)-\delta_{m}\right\}=
$$




$$
\begin{aligned}
& =\left\{\sqrt{\gamma_{m}^{h}} Z_{m}^{h}+\gamma_{m}^{h} \geq \varepsilon_{m}\left(\sqrt{\gamma_{m}^{l}} Z_{m}^{l}+\gamma_{m}^{l}\right)-\delta_{m}\right\}=\left\{\sqrt{\frac{\gamma_{m}^{h}}{\gamma_{m}^{l}}} Z_{m}^{h}+\frac{\gamma_{m}^{h}}{\gamma_{m}^{l}} \sqrt{\gamma_{m}^{l}} \geq \varepsilon_{m}\left(Z_{m}^{l}+\sqrt{\gamma_{m}^{l}}\right)-\frac{\delta_{m}}{\sqrt{\gamma_{m}^{l}}}\right\}= \\
& =\left\{\sqrt{\frac{\gamma_{m}^{h}}{\gamma_{m}^{l}}} Z_{m}^{h}+\left(\frac{\gamma_{m}^{h}}{\gamma_{m}^{l}}-\varepsilon_{m}\right) \sqrt{\gamma_{m}^{l}} \geq \varepsilon_{m} Z_{m}^{l}-\frac{\delta_{m}}{\sqrt{\gamma_{m}^{l}}}\right\}=\left\{\sqrt{\frac{\gamma_{m}^{h}}{\gamma_{m}^{l}}} Z_{m}^{h}-\varepsilon_{m} Z_{m}^{l} \geq-\left(\frac{\gamma_{m}^{h}}{\gamma_{m}^{l}}-\varepsilon_{m}\right) \sqrt{\gamma_{m}^{l}}-\frac{\delta_{m}}{\sqrt{\gamma_{m}^{l}}}\right\}= \\
& =\left\{\varepsilon_{m} Z_{m}^{l}-\sqrt{\frac{\gamma_{m}^{h}}{\gamma_{m}^{l}}} Z_{m}^{h} \leq\left(\frac{\gamma_{m}^{h}}{\gamma_{m}^{l}}-\varepsilon_{m}\right) \sqrt{\gamma_{m}^{l}}+\frac{\delta_{m}}{\sqrt{\gamma_{m}^{l}}}\right\} .
\end{aligned}
$$

Now let $\hat{Z}_{m}=\varepsilon_{m} Z_{m}^{l}-\sqrt{\frac{\gamma_{m}^{h}}{\gamma_{m}^{l}}} Z_{m}^{h}$.

Considering $\hat{Z}_{m}$, the event $A_{m}$ from (16) can be rewritten as

follows $\mathrm{A}_{m}=\left\{\hat{Z}_{m} \leq\left(\frac{\gamma_{m}^{h}}{\gamma_{m}^{l}}-\varepsilon_{m}\right) \sqrt{\gamma_{m}^{l}}+\frac{\delta_{m}}{\sqrt{\gamma_{m}^{l}}}\right\}$.

From (18) we have $Z_{m}^{h} \sim N(0,1)$ and $Z_{m}^{l} \sim N(0,1)$, and their linear combination will be distributed according to Normal distribution, as such $\hat{Z}_{m}$ will be distributed according to Normal distribution with parameters $N\left(0, \frac{\gamma_{m}^{h}}{\gamma_{m}^{l}}+\varepsilon_{m}^{2}\right)$, and subsequently from (19) we can finally obtain the expression

$$
\pi_{m}=P\left(\mathrm{~A}_{m}\right)=\Phi\left(\frac{\left(\frac{\gamma_{m}^{h}}{\gamma_{m}^{l}}-\varepsilon_{m}\right) \sqrt{\gamma_{m}^{l}}+\frac{\delta_{m}}{\sqrt{\gamma_{m}^{l}}}}{\sqrt{\frac{\gamma_{m}^{h}}{\gamma_{m}^{l}}+\varepsilon_{m}^{2}}}\right) .
$$

Thus formula (17) was obtained.

$$
\begin{aligned}
& \pi_{m}=\Phi\left(\frac{d_{m}}{\sqrt{K+\varepsilon_{m}^{2}}}\right), \\
& \text { where } d_{m}=\frac{\left(K-\varepsilon_{m}\right) \gamma_{m}^{l}+\delta_{m}}{\sqrt{\gamma_{m}^{l}}} \text { and } K=\frac{\gamma_{m}^{h}}{\gamma_{m}^{l}} .
\end{aligned}
$$

To check the relative error of the approximation formula (17), let's conduct an analysis for the earlier considered system with $M=100$ channels and $N=2000$ peers. The computation of $\pi_{m}$ will be conducted using method expressed in (11)-(13) and approximation formula (17).

The graph in Fig. 6 shows the values of the probability of channel satisfactory state obtained using formula (11) and approximation formula (17). It can be seen that relative error of the approximation formula (17) for channels with high popularities (channel 1 to 7 ) is zero. The biggest relative error of order $10^{-2}$ was recorded for 100-channel. As such formula (17) can be used for the computation of channel satisfactory state, where its correctness decreases as the channels' popularity decreases. 


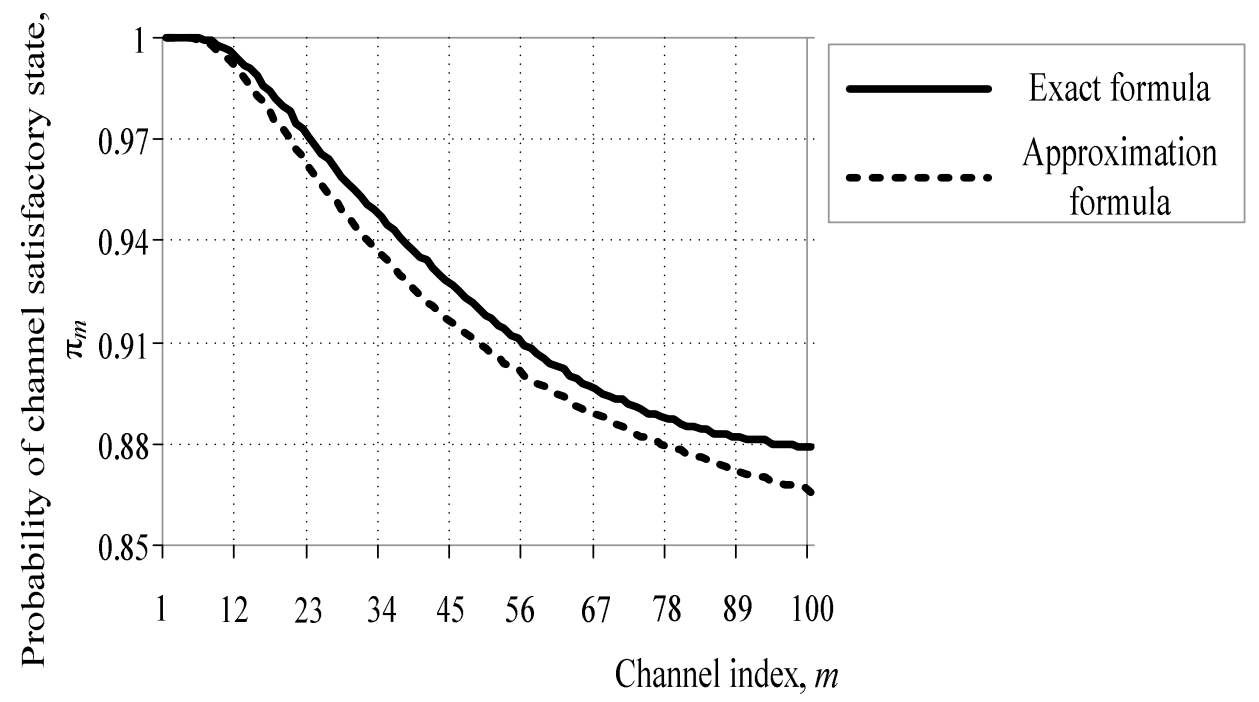

Fig.5. Exact formula vs. approximation formula

\section{CONCLUSION}

A model of P2P live streaming systems was developed based on user behaviour. One of the key QoS parameter of P2P live streaming systems was analysed i.e. probability of channel satisfactory state. An expression was obtained for the computation of the probability of channel satisfactory state, the sensitivity analysis of the QoS parameter on the number of peer with high upload bandwidth and number of peers with low upload bandwidth was carried out, it was observed that unpopular channels hardly attain satisfactory state, unlike popular channels which easily attain satisfactory state even with low number

\section{REFERENCES}

Adamu, A., and Bashir, A. J. (2015). Taxonomy of P2P networks and their associated QoS parameters. Journal of the Nigerian Association of Mathematical Physics.31, 95-100.

Kelly, F.P. (1991). Loss networks. Ann. appl. probability.1, 319-378.

Ross, K. W., Kumar, R., \& Liu, Y. (2007). Stochastic fluid theory for P2P of users with high upload bandwidth. An approximation formula for the computation of the probability of channel satisfactory state was obtained for a system with infinite number of peers, and its relative error decreases with the increase in channel popularity; however the lowest relative error of the order $10^{-2}$ was recorded for the least popular channel for a system with 100 channels.Conclusively, the results have shown that there is a need to design a framework or incentive scheme to motivate peers to contribute more of their upload bandwidth for effective functionality of P2P live streaming systems which will favour all the channels regardless of their popularities.

streaming systems. Proceedings of the IEEE INFOCOM.919-927.

Ross, K.W., Liu Y., \& Wu, D. (2010).Modeling and analysis of multi-channel P2P live video systems. Proceedings of the IEEE/ACM Transactions on Networking.1063-1072.

Shen, X., Yu, H., Buford, J.,\& Akon, M. (2010).Handbook of peer-to-peer networking. Springer 